\title{
Design of a magnetostrictive (MS) actuator
}

\author{
A. Grunwald and A. G. Olabi \\ Dublin City University, School of Mechanical and Manufacturing Engineering, Glasnevin, \\ Dublin 9, Ireland, Email: abdul.olabi@dcu.ie, artur.grunwald@dcu.ie
}

\begin{abstract}
:
Several advanced technologies are introduced in automotive applications. Higher energy density and dynamic performance are demanding new and cost effective actuator structures. Magnetostriction (MS), change in shape of materials under the influence of an external magnetic field, is one of these advanced technologies. Good understanding of specific design constrains is required to define and optimized a magnetostrictive actuator. This paper presents parametrical analysis with magnetic simulation of a magnetostrictive actuator. Proposed actuator has been designed, and the performance has been evaluated on experimental rig. Strain, elongation of the shaft, of 1000ppm at 10Amp and a blocked force over $4500 \mathrm{~N}$ has been achieved with shaft of $8 \mathrm{~mm}$ diameter, made of Terfenol-D. Furthermore, the effect of pre-stress of the Terfenol-D shaft has been evaluated experimentally. The study shows that excellent features can be obtained by magnetostrictive materials for many advanced applications.
\end{abstract}

Keywords: Magnetostriction, Actuator, Terfenol-D, FEM 


\section{Introduction of magnetostrictive (“MS”) technology}

Magnetostriction ("MS") is the change in shape of materials under the influence of an external magnetic field. The magnetostrictive effect was first described in the $19^{\text {th }}$ century (1842) by an English physicist James Joule. He observed that a sample of ferromagnetic material, i.e. iron, changes its length in the presence of a magnetic field. Joule actually observed a material with negative magnetostriction, but since that time materials with positive magnetostriction have been discovered. The causes of magnetostriction are similar for both types of material. This change in length is the result of the rotation of small magnetic domains. This rotation and re-orientation causes internal strains in the material structure. The strains in the structure lead to the stretching, in the case of positive magnetostriction, of the material in the direction of the magnetic field. During this stretching process the cross-section is reduced in a way that the volume is kept nearly constant. The size of the volume change is so small that it can be neglected under normal operating conditions. Applying a stronger field leads to stronger and more definite re-orientation of more and more domains in the direction of magnetic field. When all the magnetic domains have become aligned with the magnetic field the saturation point has been achieved. Fig.1 shows the idealized behavior of length change versus applied magnetic field. When a magnetic field is established in the opposite direction, the field is understood to be negative, but the negative field produces the same elongation in the magnetostrictive material, as a positive field would. The shape of the curve is reminiscent of a butterfly and so the curves are referred as butterfly curves. The physical background for the re-orientation of magnetic domains is depicted with some simplification schematically in Fig. 2. In the region between 0 and 1, where the applied magnetic field is small, the magnetic domains show almost no common orientation pattern. Depending on how the material was formed there may be a small amount of a common orientation pattern, which would show itself as a permanent magnet bias. The resulting strain depends very much on how homogeneous is the base structure of the magnetostrictive material and the material formulation. In the region 1-2 ideally there should be an almost linear relationship between strain and magnetic field. Because the relationship is a simple one, it is easier to predict the behavior of the material and so most devices are designed to operate in this region. Beyond point 2, the relationship becomes non-linear again as a result of the fact that most of the magnetic domains have become aligned with the magnetic field direction. At point 3 there is a saturation effect, which prevents further strain increase. With pre-stress and magnetic bias the strain capability could be optimized. The behavior of the magnetostrictive materials in various 
applications is complex, because the changing conditions during operation cause changes in material properties. The maximum useful magnetoelastic strain is one of the key parameters defining the resulting mechanical output in the case of a magnetostrictive actuator. In comparison with other magnetostrictive materials Terfenol-D $\left(\mathrm{Tb}_{0.3} \mathrm{D}_{0.7} \mathrm{Fe}_{1.9}\right)$ shows a good trade-off between high strain and high Curie temperature. The alloy formulation is known as Terfenol-D, where "Ter" is from Terbium, "Fe" is the chemical symbol for iron, "NOL" is derived from Naval Ordnance Laboratory and "D” is for Dysprosium (Ter+Fe+Nol-D). Magnetostriction only occurs in a material at temperatures below the Curie temperature, but often the Curie temperature is below the temperature of the environment and this causes the magnetostriction effect to have little practical value. Development of further alternative magnetostrictive materials is on going [1,2 and 3]. Table 1 compares typical strains for various magnetostrictive materials. In case a shaft is made of magnetostrictive material, i.e. Terfenol-D, magnetic field along the shaft axle will cause axial elongation. The maximal strain can be obtained when the crystallographic direction of the material structure is perfectly aligned along the rod axis. Some anisotropy in crystallographic material structure, cased i.e. by available manufacturing process, has to be accepted for high volume production. The elongation of the "MS"-shaft is proportional to the applied magnetic field. A higher magnetic field leads to larger elongation. Without the magnetic field the shape of the magnetostrictive material reverse to the original. The magnetostriction is a reversible feature. This unique feature from magnetostrictive material could be used for an actuator device. The "MS"technology offers an attractive controllability with high power density.

\section{Terfenol-D Shaft Analytical Calculations}

Typical actuator layouts, with their advantages and disadvantages, are depicted in Table 2 schematically. For a simple, cost-effective actuator with a high energy density the TC \& TCM configurations should be given priority. Finally the required functionality, cost level and available packaging can have an influence also on the best actuator configuration.

Fig. 3 depicts a cross-section of the actuation hardware based on magnetostrictive technology. A piezo force sensor has been integrated into the design to enable accurate and dynamic measurement of axial force. When the Terfenol-D shaft expands, it pushes against a pilot piston, which is in contact with the fluid via the hydraulic piston. This actuator in combination with a valve structure could be used as a hydraulic pump. Based on the information provided by the Terfenol-D shaft manufacturer the Young's modulus varies 
between $25 \mathrm{GPa}$ and $35 \mathrm{GPa}$. Fig. 4 shows the specification of the delivered Terfenol-D shaft. The shown data have been used as first reference for the parameter calculation.

The magnetostrictive strain for the experimental part has been specified to $800 \mathrm{ppm}$ up to 1200ppm. Fig. 6 shows the relationship between achievable operational force and both performance parameters, the upper limit of the magnetostrictive strain that can be realized and the Young's modulus of the material under these conditions. Equation (1) describes these tendencies mathematically.

$$
F_{B}^{H}=A \cdot E^{H} \cdot \lambda_{\max }^{H}
$$

There is an axial force which refers to the maximum amount of magnetostrictive strain that can be applied to a sample of Terfenol D. This occurs at very high magnetic filed strengths (at $\left.\lambda_{\max }\right)$. According to this relationship the blocked force $\mathrm{F}^{\mathrm{H}}$ is proportional to the Young's modulus and the maximal strain at an applied magnetic field. However, there also an axial force can be calculated for each value of magnetic field strength. A set of parametric calculations has been prepared in order to create a basis for the component design. MathcadProfessional-Software has been used for the following calculations. This calculation has been created considering the received specification from the manufacturer of the Terfenol-D shaft (Etrema Inc.). Expected performance and packaging leads to a solid Terfenol-D shaft with diameter of $8 \mathrm{~mm}$, and the shaft length of about $68 \mathrm{~mm}$.

\section{Optimization of the actuator performance}

All above described material properties have highly variable characteristics due to the changing conditions during an operational cycle. To achieve the desirable functionality with accuracy, good controllability and high power density, a number of additional arrangements were required to optimize the performance of a Terfenol-D transducer. One of the typical optimizing arrangements is to create a magnetic field with a permanent bias using permanent magnets, in order to operate in a region of the performance curve where the slope is at a maximum value. Above discussed condition is shown in Fig. 6. The level of premagnetization depends on the transducer design and is usually in a range from some $10 \mathrm{kA} / \mathrm{m}$ up to $100 \mathrm{kA} / \mathrm{m}$. In addition the use of magnetic bias with permanent magnets allows the transducer to be driven using bipolar current signals, thereby reducing copper losses [7,8 and 20]. The other method of optimization is to apply a defined mechanical pre-stress which can cause rotations of magnetic moments in a way that they are aligned perpendicularly to this 
applied stress. In this condition the smallest increase in the applied magnetic field will produce the greatest magnetostrictive strain, and this effect is confirmed by experiment. The required mechanical pre-stress is not very large. It can be observed that larger magnetostrictive strains can be produced by the same magnetic field strengths when the prestress is increased. This is illustrated for Terfenol-D in Fig.7.

For larger pre-stress values, another effect is observed. The energy required to overcome the pre-stress when producing a positive magnetostrictive strain becomes the dominant factor, with the result that magnetostrictive strain becomes smaller for very large values of pre-stress. There is therefore a pre-stress value where the magnetostrictive strain reaches a maximum. The optimal pre-stress is the stress which causes the magnetic moments to be aligned predominantly perpendicular to the rod's longitudinal axis without introducing so much load that the work required to overcome it becomes overwhelming [10]. The butterfly curves, showing strain versus applied magnetic field at various pre-stresses, as illustrated above, are typically used to quantify the optimal pre-stress. The strain capability of a Terfenol-D element in a transducer can be increased by factor $\sim 3$ as a result of the correct pre-stress selection. The pre-stress is applied using a spring. There is interdependence between the choice of magnetic bias and mechanical pre-stress, with one factor having an effect on the other. The choice of magnetic bias and mechanical pre-stress is investigated in several studies. A general overview of this effect is given in reference articles [9, 10, 12, 13 and 14]. Furthermore, the impact of cyclic stress on Terfenol-D on durability has to be taken into account; some information could be viewed in reference article [15]. Further optimization, linked to the same physical background, is possible by choosing the best manufacturing method [16] or annealing process [17] to improve the material texture, which has an effect on the direction of the magnetic moments.

\section{The Magnetic circuitry for the magnetostrictive actuator}

A coil in an appropriate ferromagnetic housing is defined as source of the magnetic field. The chosen diameter of the Terfenol-D shaft is $8 \mathrm{~mm}$ and the length is about $68 \mathrm{~mm}$. This selection has been based on the results of the parametric calculations. The magnetic field is result of electric power flow, current I [Amp] and voltage U [V], through the actuator coil. The coil is wound around the Terfenol-D shaft and the magnetic field is therefore parallel to the axis of the rod. Fig. 8 depicts the TC (see Table 2) layout of the actuator. Since not only the coil is involved in the magnetic circuit other ferromagnetic components like housing, Terfenol-D 
shaft and inserts have to be considered. Fig. 9 presents the main magnetic path through the magnetostrictive actuator. A moderate magnetic field strength, $\mathrm{H}(\mathrm{A} / \mathrm{m})$ is required to achieve the expected performance. Fig. 10 shows the strain capability of applied magnetic field intensity $(\mathrm{A} / \mathrm{m})$ from Terfenol-D material. The depicted data were taken from the supplier specification for the Terfenol-D shaft at pre-stress about 6.9MPa. The estimated linear range is up to $1200 \mathrm{ppm}$. The maximal targeting strain range, due to near linear relationship to the applied field, is up to the level of $1200 \mathrm{ppm}$ is the preferred dynamic operational strain for the actuator. It is common for layouts, involving Terfenol-D actuators where the magnetic flux should be guided and focused in the region of the active material. This is required to maximize the magnetic field energy and minimize the energy losses in the region where the magnetic field is not needed. The frequency range has also to be considered in order to enable the functionality of the actuation system. A nonlinear time harmonic simulation method will be used to determine and optimize the actuator performance. The parameter calculations from this study related to the operational frequency would be used to set up the first set of parameters for the simulation.

According to the Ampere's Law the relationship between the current (A) and the magnetic field density $(\mathrm{A} / \mathrm{m})$ could be formulated as:

$$
\oint H d l=N \cdot I
$$

In Fig. 9 there are six designated sections of component through which the magnetic flux passes. Therefore:

$$
\oint H d l_{G M}=N_{G M M} \cdot I_{G M}=H_{1} \cdot l_{1}+H_{2} \cdot l_{2}+\cdots+H_{6} \cdot l_{6}
$$

It is important to include all six terms in order to estimate the required total magnetomotive force, and so the return path of the magnetic flux through steel components must also be considered. For each section there is a length of the magnetic path, 1, and a value for the magnetic field strength, $\mathrm{H}$. The length is fixed by the geometry of the system, but the value of $\mathrm{H}$ must be determined by making use of the magnetic properties of the material. For the housing of the actuator the low carbon steel, $\mathrm{Ck} 15$, has been used. The magnetic properties of Ck15 are shown in Fig. 11.

The magnetic properties of Terfenol-D at various pre-stress levels have been provided by the Terfenol-D supplier. Fig. 12 depicts the B-H characteristic of the preferred pre-stress about 7.2MPa. In order to enable the actuation mechanism to work at high dynamic level, it is required to minimize the amount of the ferromagnetic steel in the magnetic circuit. For a 
magnetic circuit the total flux, $\phi$, remains constant. The flux is related to the flux density, $\mathrm{B}$, and is equal to the flux density multiplied by the integral of cross-sectional area [18]:

$$
\phi=\int B d A
$$

Where this area is constant throughout the magnetic pathway, the integral of cross-sectional area is equal to the area. Since the flux is constant, the product of flux density and crosssectional area must be constant for each material:

$$
\begin{gathered}
\phi_{\text {Terfenol }}=\phi_{\text {steel }} \\
B_{\text {Terfenol }} \cdot A_{\text {Terfenol }}=B_{\text {steel }} \cdot A_{\text {steel }}
\end{gathered}
$$

The flux density B (Tesla) depends on the properties of the medium and specially the relative $\mu \mathrm{r}(-)$ and absolute permeability $\mu$. They are sometimes written in the form:

$$
\begin{gathered}
B_{\text {steel }}=\mu_{0} \cdot \mu_{\text {steel }} \cdot H_{\text {steel }} \\
B_{\text {Terfenol }}=\mu_{0} \cdot \mu_{\text {Terfenol }} \cdot H_{\text {Terfenol }}
\end{gathered}
$$

where $\mu_{0}$, the permeability of free space is constant, and $\mu_{\mathrm{r}}$, the relative permeability is another variable with different values for each value of magnetic field strength and for each material. The reluctance, $\mathfrak{R}$, is an analog to the resistance in the electric circuit. For the calculation of the reluctance the following equation could be stated:

$$
\Re=\frac{M M F}{\phi}=\frac{N \cdot I}{\phi}
$$

The reluctance is sometimes written in the form:

$$
\mathfrak{R}=\frac{l}{\mu_{0} \cdot \mu_{R} A}
$$

The total system reluctance can be calculated as follows:

$$
\mathfrak{R}_{\text {total }}=\mathfrak{R} 1+\mathfrak{R} 2+\mathfrak{R} 3+\cdots+\mathfrak{R} n
$$

Ohm's law for magnetic circuit could be formed as:

$$
M M F=\phi \cdot \Re
$$

In the magnetic path through an assembly, components specific reluctance has to be considered separately:

$$
M M F=\phi \cdot(\mathfrak{R} 1+\mathfrak{R} 2+\mathfrak{R} 3+\cdots+\mathfrak{R} n)
$$


The inductance (Weber-turns per Ampere) of the magnetic circuit is defined as:

$$
L=\frac{N^{2}}{\Re_{\text {total }}}
$$

Current demand in the range of 10A would be a good trade off between low current and good performance requirements for of automotive applications. Due to combination of equations (10) with (11), considering equations (6) to (8), following equation could be introduced:

$$
N=\frac{\mathfrak{R}_{\text {total }} \mu_{0} \cdot \mu_{\text {Terfenol }} \cdot H_{\text {Terfenol }} A_{\text {Terfenol }}}{I}
$$

It is now possible to follow the procedure to calculate the required number of turns to achieve the required magnetic field strength. For the parametric calculation, to determine the number turns, the relative permeability of Ck15 and Terfenol-D has been set constant. These constants, Ck15 and Terfenol-D, have been set to average value for specific material based on measurements. Fig. 13 depicts the results from the calculation for the required number of turns. These calculations are prepared as the first input for simulation of the magnetic circuit. The nonlinear B-H characteristic and the real, not constant permeability of each material will be used in the magnetic field simulation. The proposed approach, outlined above, was also used to calculate the reluctance of the circuit; it is $60^{\mathrm{x}} 10^{6} \mathrm{Henry}^{-1}$. For above parametrical calculation it was necessary to perform a trial and error series of operations to find out how many turns the coil needs to have in order to provide the required field strength and how much space this coil will need. To perform these calculations, layout geometries had to be assumed initially, i.e. the outer diameter of the housing, and then tuned to the optimum. Finally, magnetic field simulation has been used to confirm the magnetic field calculations. Presented calculation results have been used as the initial input for the magnetic simulation software for further optimization. In the following calculation it was necessary to perform a trial and error series of operations to find out how many turns the coil needs in order to provide the required field strength and how much space this coil will need. The proposed approach with the calculation of the reluctance enables to indicate the dynamic capability of the proposed arrangement. Fig. 14 shows the cross section of an air coil. The bobbin of the coil is made of aluminum and does not affect the magnetic field. Each strand of the copper wire is coated with an insulating layer to avoid electrical short circuits. The insulated wire is about 10\% larger than the un-insulated wire. The overall resistance of the copper wire in the coil can be calculated if the geometry, length and cross-sectional area, are known and the resistivity of copper is known. 


$$
R_{d c}=\frac{\delta \cdot l_{\text {wire }}}{A_{\text {wire }}}
$$

The resistivity of copper, $\delta$ with units, is defined by:

$$
\delta_{c}=0.01786 \cdot \frac{\Omega \cdot \mathrm{mm}^{2}}{m}
$$

There is also a variation of the resistivity of copper with temperature, and this also must be taken into consideration. Over the temperature range of interest:

$$
R_{\theta}=R_{20} \cdot\left(1+\alpha_{20} \cdot\left(\theta-20^{\circ} C\right)\right)
$$

where $\theta$ is the temperature in degrees centigrade and the temperature constant, at $20^{\circ} \mathrm{C}$ with units $1 /{ }^{\circ} \mathrm{C}$, is defined by:

$$
\alpha_{20}=0.0039 \frac{1}{{ }^{\circ} \mathrm{C}}
$$

Fig. 15 shows the result of the parametric calculation of current density versus wire diameter. The specified current range 1-10 A has been used. Typical current density in automotive applications is lower then $7 \mathrm{~A} / \mathrm{mm}^{2}$. The current density limitation, given by wire supplier, is up to $10 \mathrm{~A} / \mathrm{mm}^{2}$. Generally, the limitation is given by thermal conditions and the isolation layer specification. It is now necessary to perform a trial and error series of operations to find out how many turns the coil needs to have in order to overcome the reluctance. Another way to calculate is the definition of required magnetomotive force. The result of both ways of calculation leads to the same coil specification. The parametrical calculation results, used to specify the coil for experimental evaluation, have been proven by non-linear magnetic field simulation. Based on above discussed analytical calculations and the results from the magnetic field simulations, specification for coils has been proposed. Fig. 16 depicts geometry and reference coil specification of magnetostrictive actuator.

\section{Magnetic field simulation results from "MS"-actuator}

In order to optimize the design of the actuator, a simulation of magnetic field, including the real magnetic properties of used material, is required. The nonlinear simulation method was used to determine and optimize the actuator and control performance. Fig. 17 shows the meshed structure of the magnetostrictive actuator. The simulation problem type has been set up to an "axisymmetric problem". For the simulation the "asymptotic boundary conditions" 
have been applied. The results of the parametric calculation for the electric circuit have been used to set up the complete model of the actuator assembly. The measured B-H characteristic for each material has been used as input for the magnetic field simulation software.

Fig. 18 shows the density of B (Tesla) related to the center line of the Terfenol-D shaft. The results shown in Fig. 18 has been obtained using FEMM software package, version 4. Based on the parametric calculations the maximal current for the actuator coil has been set up to 9 Amperes. Various types of output information could be used to evaluate the magnetic field. For the evaluation of the magnetic circuit voltage drop, flux linkage, inductance, resistance and electrical power have been calculated. All calculations have been performed with frequencies $0 \mathrm{~Hz}, 10 \mathrm{~Hz}, 50 \mathrm{~Hz}, 250 \mathrm{~Hz}, 500 \mathrm{~Hz}$ and $1000 \mathrm{~Hz}$.

Fig. 19 presents the assembly reference picture for 2D-plots for magnitude of field density B (Tesla) and magnitude of field intensity $\mathrm{H}(\mathrm{A} / \mathrm{m})$ of the Terfenol-D shaft along the defined path line marked as length. The geometry of the assembly has been verified up to the homogeneous density of the magnetic field along and across the Terfenol-D shaft has been achieved and the density level was acceptable. The initially proposed geometry, based on analytical calculation, has been optimized with the simulation tool. Several loops of simulations have been performed before the design freeze for experimental rig. At the prestress of $6.9 \mathrm{MPa}$ and the field intensity about $100000 \mathrm{~A} / \mathrm{m}$ the expected strain of the Terfenol-D shaft should be higher than 1200ppm. This result will be within the specified range and was rated as acceptable. A homogeneous distribution of the magnetic field intensity along and across the Terfenol-D shaft has been achieved and the intensity level has been rated as acceptable. The results of the magnetic field simulation have been used to set up the design freeze for prototyping. For comparison and verification reasons the "MS"-actuator assembly has been evaluated using as well the Vector Fields Software for magnetic design, called Opera. All the geometries and material data as well the boundary conditions have been kept equivalent to the FEMM model. Fig. 20 shows the density plot of B (T) related to the centre line of the Terfenol-D shaft. The results shown in Fig. 20 have been obtained using Opera software version 10.017 and results using FEMM software version 4.0.1. Fig. 21 shows the magnitude of field intensity $\mathrm{H}(\mathrm{A} / \mathrm{m})$ related to the centre line of the Terfenol-D shaft. Fig. 21 shows results which have been obtained with two different simulation systems, Opera and FEMM. Comparing the simulation results, obtained by FEMM and Opera, it is obvious that both results are in perfect coincidence. It can be noted that the free available software package FEMM offers a very simple user interface. The professional software packaging from Vector Fields is wider purpose software for electromagnetic design. Various types of solutions for the 
proposed actuation system are available: static analysis module, steady-state module and the transient analysis module.

\section{Experimental rig evaluation}

To prove feasibility of the actuation principle experimental rig parts have been produced. The magnetostrictive actuator assembly, magneto-rheological control assemblies and load assembly has been prepared for experimental evaluation. The test results have been used to confirm the analytical calculations and the magnetic field simulation and to highlight the potential of performance optimization. The experimental rig parts of the magnetostrictive actuator are shown in Fig. 22. The measured weight of the "MS"-actuator assembly is $1560 \mathrm{~g}$ and the volume is $250 \mathrm{~mm}^{3}$. The actuator assembly has been completed and used to evaluate the performance (strain capability and blocked force level) of the magnetostrictive actuator. Fig. 23 depicts the test bench layout for force and for axial displacement measurements. Fig. 24 depicts the cross section of the tested assembly with force sensor and displacement sensor. Using above shown assembly the specified performance of the actuator, based on magnetostrictive technology, has been proven on the rig test. Firstly, the effect of pre-stress has been evaluated. A piezo-sensor has been integrated into the assembly for accurate and dynamic force measurement. Recorded data versus time were current, voltage, axial force and axial displacement. An evaluation of the displacement versus current has been used to define the optimal pre-stress of Terfenol-D shaft in the assembly. Pre-stress range of 6-34Mpa (300N-1700N pre-load) has been considered in this test session. Fig. 25 to 28 show the key results from experimental evaluation of the magnetostrictive actuator at low and medium preload. The dependency of the achieved axial displacement, at low pre-load, versus electrical current is shown in Fig. 26. The dependency of the achieved axial displacement, at medium pre-load, versus electrical current is shown in Fig. 28.

The effect of pre-stress has been evaluated experimentally. The level of achieved strain has been rated as sufficient and is within the specified range. The optimal pre-load has been set to the axial force of 500-600N. At this axial force the Terfenol-D shaft is pre-stressed with 10$12 \mathrm{MPa}$ and the achievable displacement is about $0.065 \mathrm{~mm}$. Related to the tested length of the Terfenol-D shaft, the strain is approximately $1000 \mathrm{ppm}$. This strain is within the specified strain range of the "MS"-shaft. The dependency of the achieved axial displacement versus electrical current is shown in Fig. 29. In Fig. 29 the pre-load effect is shown as parameter. 
The evaluation of the frequency impact on the actuator performance strain capability has been carry out in the next test. Further, the butterfly-curves with sinusoidal power input have been measured to confirm symmetric behavior of the Terfenol-D. During this test procedure the frequency dependency has been evaluated. In Fig. 30 to 32 show the displacement versus current at various frequencies and constant pre-load. Generally, butterfly-curves and the symmetric behavior of the Terfenol-D actuator have been conformed. Some test equipment limitations (available power stage) and noise from the available displacement sensor have destroyed the symmetric behavior. To evaluate the Terfenol-D actuator capability regarding the maximal axial force, the disc spring has been replaced by a washer. Considering the maximal compressive stress of the material two additional levels of pre-load have been tested. At the medium pre-load level of $1.7 \mathrm{kN}$ a clear butterfly curve could be observed and strain capability about $600 \mathrm{ppm}$ (shaft elongation of $0.037 \mathrm{~mm}$ ) has been measured. The measuring data are shown in Fig. 33. With the increase of the pre-load force the actuator comes into the blocked force mode and the butterfly-curve is hardly visible. At the pre-load of approximately $4.5 \mathrm{kN}$ the useable strain is very limited and could not be used any more as considerable displacement for a pump or other actuator principles. Fig. 34 depicts the hardly recognized butterfly-curves at blocked force level at which the strain capability is not significant any more. The achieved results of the magnetostrictive actuator: acceptable displacement, good functionality at various frequencies and high blocked force level has been found as sufficient and in line with theoretical performance calculations.

\section{Conclusions}

High power density and simple design of the magnetostrictive actuator has been evaluated and optimized. A literature survey for "MS"-technology presented in article [19] has been extended with analytical calculations, magnetic field simulations and experimental evaluation. Based on calculations and simulation results, the design of an "MS"-actuator has been proposed. Experimental rig has been built and the specified performance has been proven with experimental results. Acceptable magnetostrictive strain and a high blocked force have been achieved. The key experimental results, related to "MS"-actuator, are summarized in Table 3. Based on achieved results it could be stated that magnetostrictive technology offers big potential for various automotive applications. Summarizing it can be stated that "MS"technology, with the smart materials like Terfenol-D, offer very attractive and valuable features. The precise small motions with high energy density and fast control response could 
be applied as well in some automotive and aerospace applications. Further extension of the "know-how" related to smart technologies, like "MS", will enable researchers and engineers to create and to establish new products in various industries. Development of new pplications with smart dynamic motions at high energy density is proposed as the next step. 


\section{Reference list}

[1] S. Guruswamy, N. Srisukhumbowornachai, A.E. Clark, J.B. Restroff, M. Wun-Fogle, Strong, Ductile and Low-Field-Magnetostrictive Alloys Based on Fe-Ga, Scripta material 43 (2000), pp. 239-244

[2] Z.D. Zhang, B.W. Wang, Z.J. Guo, S.C. Busbridge, A.R. Piercy, Dynamic magnetic and magnetoelastic properties of epoxy-TbFe2 composites, Journal of Magnetism and Magnetic Materials 239 (2002), pp. 554-556

[3] Energen Inc., Magnetic “Smart” Materials, Company, information available on http://www.EnergenInc.com

[4] ETREMA Products Inc., Terfenol-D Magnetostrictive Actuator Information, Specifications, Public domain information, www.terfenoltruth.com, www.etrema.com

[5] C.H. Joshi, Compact Magnetostrictive Actuators and Linear Motors, Conference Actuator 2000, Bremen, Germany June 2000, Conference handout paper

[6] H.P.R. Frederikse, Properties of magnetic materials, www.nmsl.chem.ccu.edu.tw.pdf, CRC Press LLC, 2000

[7] H. Janocha (ed.), F. Claeyssen, Adaptronics and Smart Structures, Springer Verlag, ISBN 3-540-61484-2, Germany 1999, pp. 124-143

[8] H. Janocha (ed.), Actuators, Springer Verlag, ISBN 3-540-61564-4, Germany 2004, pp. 277-292

[9] F.T. Calkins, M. J. Dapino, A.B. Flatau, Effect of pre-stress on the dynamic performance of a Terfenol-D transducer, 1997 SPIE's Symposium on Smart Structures and Materials, paper \# 3041-23

[10] R. Kellog, A. Flatau, Blocked force investigation of a Terfenol-D transducer, 1999 SPIE's Symposium on Smart Structures and Materials, paper \# 3668-19

[11] P.A. Bartlett, S.J. Eaton, J. Gore, High-power, low frequency magnetostrictive actuation for anti-vibration applications, Sensors and Actuators A91 (2001), pp. 133-136 [12] A.B. Flatau, F.T. Calkins, M.J. Dapino, Transducer based Terfenol-D Property Measurement, SPIE 1996, Proceedings on Smart Structures and Integrated Systems, paper \#67, Vol. 2717, 2/96

[13] L.E. Faidley, B.J. Lund, A. Flatau, F.T. Calkins, Terfenol-D elasto-magnetic properties under varied operating conditions using hysteresis loop analysis, 1998 SPIE's Symposium on Smart Structures and Materials \# 3329-92 
[14] F.T. Calkins, A.B. Flatau, Transducer based on measurements of Terfenol-D

material properties, 1996 SPIE's Symposium on Smart Structures and Materials \# 2717-67

[15] K. Prajapati, R.D. Greenough and A.Wharton, Effect of Cyclic Stress on Terfenol - D, Journal: IEEE Transactions on Magnetics Vol. 32 No.5 September 1996

[16] J.D. Snodgrass, O.D. McMasters, Optimized Terfenol-D manufacturing processes, Journal of Alloys and Compounds 258, 1997, pp. 24-29

[17] W.J. Park, Z.H. Lee, J.C. Kim, B.J. Ye, Marcrosegragation in Bridgman growth of Terfenol-D and effects of annealing, Journal of Crystal Growth 212 (2000), pp. 283-290

[18] A.E. Fitzgerald, Ch. Kingsley, St. D. Umans, Electric Machinery, Mc Graw Hill, 2003, ISBN 007366009-4, pp. 1-30

[19] A. G. Olabi, A. Grunwald, Design and optimization of magnetostrictive actuator, Materials \& Design, Vol 29, Issue 2, 2008, pp. 469-483.

[20] G. Engdahl (ed.), Handbook of Giant Magnetostrictive Materials, Royal Institute of Technology, Stockholm, Sweden, ISBN 012238640, pp. 1-373 